\title{
Research on Influencing Factors of Sport Injury of Competitive Aerobics Athletes
}

\author{
Zou Wenjun ${ }^{1}$, Zeng Yunjing ${ }^{2}$ \\ ${ }^{1}$ Institute of Technology East China Jiaotong University, Nanchang, Jiangxi, China \\ ${ }^{2}$ Beijing Sport University, Beijing, China
}

Keywords: competitive aerobics; sport injury; athlete; influencing factor

\begin{abstract}
In recent years, the excellent achievements of Chinese competitive aerobics athletes in international competitions have proved the level of sports of competitive aerobics athletes. However, sport injuries occur during the training and competition of athletes from time to time, which will affect the athletes' performance, even directly lead to the diseases, and ultimately affect the athletes' lifelong health. It is imperative to take corresponding measures to reduce the probability of competitive aerobics sport injuries. This paper analyzes the influencing factors and prevention measures of sport injuries of competitive aerobics athletes.
\end{abstract}

\section{Research Object, Research Content and Methodology}

Research object, research content and research methodology are analyzed in this section.

\subsection{Research Object}

The main object of this research is the competitive aerobics athletes in colleges and universities in Jiangxi Province, including 58 male athletes and 42 female athletes.

\subsection{Research Content}

The main content of this research is mainly the sport injuries that athletes have experienced in competitive aerobics training and competition.

\subsection{Research Methodology}

The main research methodology of this research are presented. Firstly, the relevant competitive aerobics sport injury survey form is designed, and ensured to be as objective and comprehensive as possible with the help of relevant professionals. Secondly, the questionnaire is issued to 100 athletes surveyed and 100 copies are collected. Thirdly, statistics and analysis are conducted on the contents of the questionnaire survey.

\section{Research Results and Analysis}

Sport injury and categories, sport injury part, sport injury degree, technical grade of research object, the time and factor of sport injury are analyzed in this section.

\subsection{Sport injury categories}

According to relevant data, among the 100 athletes surveyed, 84 athletes had sport injuries, and the probability of sport injuries is $84 \%$. There are 10 sport injuries in total, including muscle ligament injury, joint damage and its lesion, tenosynovitis, meniscus injury, fracture, periostitis, bursitis, contusion, patella chondropathy, knee extension aponeurosis fibrosis. Among them, 100 people had muscle ligament injury, and the constituent ratio is $100 \%$; joint damage and lesion occurred in 46 people, accounting for 46\%; 73 people had tenosynovitis, accounting for $73 \%$; 15 people had meniscus injury, accounting for 15\%; 5 people had fracture, accounting for 5\%; 16 people had periostitis, accounting for 16\%; 8 people had bursitis, accounting for $8 \%$; 9 people had contusion, accounting for $9 \%$; 6 people had patella chondropathy, accounting for $6 \%$; 4 people had 
knee extension aponeurosis fibrosis, accounting for $2 \%$.

\subsection{Sport injury part}

The main sport injury parts of athletes include wrists, ankles and feet, calves, knees, waist, shoulders, thighs, elbow joints, neck, etc., and wrists, ankles and feet, waist, shoulders, knees are most common sport injury parts, accounting for $62 \%$, 30\%, 29\%, 27\%, and 26\%, respectively.

\subsection{Sport injury degree}

The degree of sport injury of athletes can be divided into mild injury, moderate injury and severe injury, accounting for $20 \%, 12 \%$ and $4 \%$ respectively.

\subsection{Technical grade of research object}

The technical grade of research subject can be divided into athletes according to the national elite athletes, national level-1 athletes, national level-2 athletes, and athletes below level-2, among which the number of national elite athletes is 16 , the number of athletes at the national level- 1 is 32 , the number of national level-2 athletes is 32, and the number of athletes below national level-2 is 20 , and the sport injury rates of athletes of different grades are $62.5 \%, 87.5 \%, 78.1 \%$ and $75 \%$ respectively.

\subsection{The time of sport injury}

The time of sport injury can be divided into January-March, April-June, July-September, and October-December, accounting for 37\%, 26\%, 23\%, and 14\%, respectively.

\subsection{The factor of sport injury}

By comprehensive analysis, it can be concluded that there are mainly eight factors of sport injury of competitive aerobics athletes, namely, site factors, psychological factors, technical factors, local overload factors, sports fatigue factors, and insufficient preparation activities, and assault difficulty action factors.

\section{Analysis on Influencing Factors of Sport Injury of Competitive Aerobics Athletes}

Through the analysis of the results, it can be seen that the factors affecting the sport injury of competitive aerobics athletes are in many aspects. The detailed analysis from potential factors and direct factors of sport injuries of competitive athletes is presented.

\subsection{Potential factors of sport injury of competitive aerobics athletes}

The potential factors is analyzed from characteristics and sport rules of competitive aerobics sport events.

\subsubsection{Characteristics of competitive aerobics sport events}

The competitive aerobics is a skill sports characterized by its flexibility as the basis, strength as the core and the endurance as the guarantee. The requirements for athletes are very high, mainly because athletes need to complete difficult dynamic and static movements in a short period of time, and the combination of these movements is very complicated and difficult. At the same time, the high frequency and large amplitude are the main features of these movements. Therefore, the physical aerobics athletes are under great pressure during the exercise process, and injuries will occur directly if they are careless. Through the research, it can be seen that competitive aerobics is an event that injury is easy to occur.

\subsubsection{Rules of competitive aerobics sport events}

The rules of competitive aerobics stipulate that the athlete's complete set of movements must reflect four types of movements, push-up, free falls, leg circles and cuts; support and change; jumping; flexibility and change, and the total difficulty of the four movements is required to be controlled within 12 . For the first two movements, the movement needs to be gradually mastered in 
the continuous practice, and in this exercise process, the injury rate of wrist is the highest, reaching $25 \%$. The latter two types of movements need the constant improving of the flexibility of the body. Therefore, in the practice of these two types of movements, the muscle and ligament lesion of athletes occur very frequently, and the incidence rate can reach $35.3 \%$.

\subsection{Direct factors of sport injury of competitive aerobics athletes}

Direct factors of sport injury of competitive aerobics athletes include four aspects, sport fatigue, site factors, technical factors and other factors, which is analyzed in detail.

\subsubsection{Sport fatigue}

The so-called sport fatigue means that if the exercise time exceeds a certain range, then the various functions and organs of the body cannot maintain the original working ability, and the working ability will decrease. The main cause of sport fatigue of competitive aerobics is deficient oxygen and muscle fatigue. Because in competitive aerobics, athletes need to complete large-amplitude, high-intensity and high-efficiency movements in a short period of time. In the process of training, the training content is easily too large, and the body function is insufficient, thus in completing the second half of the movement, athletes are very prone to lack physical strength, resulting in a decline in the quality of the movement, movement errors, etc., and finally there will be injury.

\subsubsection{Site factor}

The movement of competitive aerobics is very rich, mainly including skipping, jumping, circling, supporting, transiting and so on, because the requirements for the sports venue are also quite high. Once the sports venue is too slippery, too hard, etc., it will directly affect the actual movement of athletes, which will lead to the occurrence of injury.

\subsubsection{Technical factor}

As an immature competitive sport, the training methods of competitive aerobics are not perfect at present, especially the standardization of technical movements, which cannot be completely guaranteed in the actual training process. According to relevant survey, technical factors directly led to the $14.5 \%$ of physical injury of competitive aerobics athletes.

\subsubsection{Other factors}

According to relevant survey, during the course of sports, competitive aerobics athletes have many injuries due to other factors, such as sudden accidents, mutual contact collisions between athletes, inappropriate sportswear, etc. Although the probability of physical injury because of these factors is small, it indeed happened. At the same time, athletes' own psychological quality can directly affect the occurrence of physical injury during the movement.

In short, it can be seen that for competitive aerobics, the factors of sport injuries that may occur are various, and not every factor can be avoided $100 \%$, but the author believes it is also necessary to take corresponding measures and prevention measures according to the influencing factors of sport injuries of competitive aerobics athletes, as far as possible to reduce the probability of sport injuries during sports, which is meaningful for the personal health and the future development of competitive aerobics athletes.

\section{Prevention Measures of Sport Injury of Competitive Aerobics Athletes}

Prevention measures of sport injury of competitive aerobics athletes are analyzed in six aspects, specifically, to conduct sufficient warm-up and standardize movement technology; to strengthen the physical stamina training of athletes and enhance physical stamina reserve; to reasonably arrange training content and exercise load; to pay attention to the training and recovery and relaxation after competition; to strengthen the training of psychological quality of athletes; to strengthen medical supervision and improve the self-protection awareness of athletes. 


\subsection{To conduct sufficient warm-up and standardize movement technology}

In competitive aerobics, the lack of warm-up will directly cause sport injuries. Therefore, in the process of sports, it is very important to pay attention to the warm-up before the exercise. In addition, the movement technology that meets the standards is more effective in avoiding sport injuries, which shall be emphasized by both coaches and athletes.

\subsection{To strengthen the physical stamina training of athletes and enhance physical stamina reserve}

Aerobics is a difficult and high-intensity sports event. If the athlete's own exercise intensity and physical quality are not enough, it will inevitably lead to sport injuries. At this time, it is necessary to strengthen the training of athletes' physical stamina to adapt to the requirements of competitive aerobics for athletes. In view of how to strengthen athletes' physical training, the author believes that the coach should customize a specific physical exercise program for different athletes, and ultimately enhance athletes' physical stamina reserve.

\subsection{To reasonably arrange training content and exercise load}

Only by formulating scientific and reasonable training content according to the athlete's own characteristics can the training play the most important role and significance, and can also effectively avoid the mode of concentrating on one thing. In addition, the exercise load, and the combination of high intensity and low intensity are appropriate to avoid the high-load exercise, and the training needs to be a step-by-step process. In the process of training, it is also necessary to realize the importance of increasing basic training and assisted training, and also to pay attention to the athlete's physical problems and the combination of exercise and rest, and finally, to avoid training and exercise with sickness and injury.

\subsection{To pay attention to the training and recovery and relaxation after competition}

Most of competitive aerobics are anaerobic exercise, so there will be lactic acid accumulation after exercise or training. At this time, it can only be improved by traction. Then, after the exercise, it is also necessary to restore the athlete's physical stamina from both diet and sleep. At the same time, massage and psychological relaxation therapy can also be applied to relieve fatigue and ensure that future training can proceed smoothly.

\subsection{To strengthen the training of psychological quality of athletes}

For competitive aerobics, it is characterized by high intensity, high frequency and many movements, so athletes are also very likely to cause movement errors due to poor psychological quality during the exercise process, which eventually causes injury. At this time, it is necessary to strengthen the training of athletes' psychological quality. Generally speaking, the psychological quality of athletes can be improved from both theoretical and practical aspects, and ensures that athletes will not suffer from sport injuries due to poor psychological quality. In addition, to improve the psychological quality of athletes can effectively help athletes improve their athletic performance and enable them to play a higher level in the process of sports.

\subsection{To strengthen medical supervision and improve the self-protection awareness of athletes}

Finally, to prevent sport injuries by strengthening medical supervision and improving athletes' awareness of self-protection is mainly to carry out systematic and comprehensive medical supervision on the training content and movement of athletes, so as to more comprehensively understand the sports skills of athletes and the changes in the technical level of aerobics, which can not only provide training content for athletes in addition to certain help, but also protect the health of athletes in this process. In addition, athletes themselves need to raise their awareness of self-protection. Once an abnormality occurs in a certain part of the body, it is necessary to carry out timely treatment to avoid sport injury. At the same time, athletes also need to pay close attention to the weather, clothing, site and other factors to ensure that these aspects are normal to prevent sport injuries. 


\section{Conclusion}

Through the analysis, conclusions can be made in three aspects. First of all, the probability of sport injury of competitive aerobics athletes is generally higher; secondly, the main body parts of sport injuries include wrists, ankles, shoulders and waist, and the common diseases are muscle ligament lesion, joint damage, etc.; thirdly, the main factors of sport injury of competitive aerobics athletes are various, such as site factors, psychological factors, technical factors and so on. At the same time, there also exist inevitable potential factors, such as characteristics of competitive aerobics and the difficulty. In conclusion, this paper mainly analyzes influencing factors of sport injury of competitive aerobics athletes, and takes relevant measures to reduce the probability of sport injury to the lowest, and prevention measures from six aspects, specifically, to conduct sufficient warm-up and standardize movement technology; to strengthen the physical stamina training of athletes and enhance physical stamina reserve; to reasonably arrange training content and exercise load; to pay attention to the training and recovery and relaxation after competition; to strengthen the training of psychological quality of athletes; to strengthen medical supervision and improve the self-protection awareness of athletes, which can provide reference for relevant personnel.

\section{Acknowledgements}

Fund project: This paper comes from scientific and technological research project of Jiangxi Province Department of Education. Project Name: Research on Influencing Factors of Sport Injury of Competitive Aerobics Athletes. Project No. GJJ161559.

\section{References}

[1] Zheng Min. Training Methods of Difficult Competitive Calisthenics Movements [J]. Journal of Wuhan Institute of Physical Education, 2003(06).

[2] Zha Chunhua. On Function and Composition of Music in Sports Aerobics [J]. Journal of Zhejiang Normal University (Natural Sciences), 2004(02).

[3] Yuan Zhihua. On the Training of Competitive Aerobics from New Rules [J]. Journal of Chengdu Sport University, 1997(01).

[4] Li Hongling. On Training Methods of Psychological Quality of Competitive Aerobics Athletes [J]. Journal of Yunmeng, 1998(04).

[5] Zhang Huanwei, Li Zhenliang. Research on Factors Affecting the Aerobics Performance of College Students [J]. China Science and Technology Information, 2007(19).

[6] Teng Yanfeng. On College Students’ Competitive Aerobics Difficulty Movement Training Method Research [J]. Journal of Langfang Teachers University (Natural Sciences), 2011(05).

[7] Dou Dan, Chen Xiaoying. Application of Chinese Classic Dance Training System to Aerobics Training [J]. Journal of Southwest Agricultural University (Social Science Edition), 2005(01).

[8] Shen Mingyu. Research on Sport Injury of Competitive Aerobics Athletes [J]. Contemporary Sports Technology, 2015(32).

[9] Sun Xuanxuan, Zhao Lina, Liu Li, Liu Zhihong. Primary Exploration on the Performance of Adolescent Aerobics Training [J]. Journal of Beijing Sport University, 2007(S1).

[10] Fang Zhehong. Training Countermeasure of Aerobics Exercises with the Rule Changes [J]. Journal of PLA Institute of Physical Education, 2002(03). 